Military Technical College, Kobry El-Kobbah, Cairo, Egypt $9^{\text {th }}$ International Conference On Aerospace Sciences \& Aviation Technology

\title{
AUTOMATIC CLASSIFICATION OF MPSK SIGNALS IN PRESENCE OF SATELLITE CHANNEL IMPAIRMENTS
}

\author{
AZZOUZ ${ }^{*}$ E. E. and HELALY ${ }^{*}$ T. K.
}

\begin{abstract}
Satellite communications have received an increasing attention in the last three decades because of their potential service to rural areas that cannot economically be incorporated into terrestrial systems. For satellite communications, M-ary Phase Shift Keying (MPSK) schemes are widely used because they offer a good trade-off between power and bandwidth. In this paper an algorithm for automatic real time classification of MPSK with $M=2,4$, and 8 in presence of satellite channel impairments is introduced. A combination of digital signal processing and pattern recognition methods is used for solving the recognition problem. The developed algorithm has been tested by computer simulations and has proven to be reliable and robust against the expected channel impairments (interference) such as noise, fading, shadowing, and transponder non-linearity. It is found that all digital modulation types of interest have been correctly classified with a success rate $>94$ $\%$ at signal-to-interference ratio, SIR, of $10 \mathrm{~dB}$.
\end{abstract}

\section{KEY WORDS}

Signal processing, satellite communication, and modulation classification

\footnotetext{
Egyptian Armed Forces
} 


\section{Introduction}

This paper is concerned with the automatic modulation recognition of satellite communication signals. Modulation recognition is a branch of non-cooperative communication theory such as electronic warfare (EW), surveillance, and threat analysis. Also, modulation recognition is extremely important in communication intelligence (COMINT) applications. In satellite environments, there exist two serious problems in addition to noise. The first is the fading and shadowing while the second is the transponder non-linearity. Depending on the satellite orbit, in Medium Earth Orbit (MEO) and Geostationary Earth Orbit (GEO) satellites, high power is required to maintain an accepted service quality and then non-linearity of the Traveling Wave Tube Amplifier (TWTA) has to be considered. It is assumed that a well backing-off is applied to the input power to the TWTA of the transponder to reduce the effect of non-linearity. Also, for reasons of simplification the fading effect is assumed to be slow which is almost a real case. The developed algorithm in this paper utilizes the statistical pattern recognition approach for the classification of MPSK signals. This algorithm is based on the phase characteristics of the intercepted signal. These characteristics are such that for each type of MPSK a certain number phase levels are assigned.

\section{Theoretical background}

Let the modulated signal, $x(t)$, be

$$
x(t)=\operatorname{Re}\left\{A\left[e^{j\left(\omega_{c} t+\theta_{a}(t)\right)}\right]\right\}
$$

where $A$ is a constant amplitude, $\omega_{c}$ is the carrier angular frequency, $\theta_{G}(t)$ is the signal phase, and $a=1,2, \ldots, \log _{2} \mathrm{M}, \mathrm{M}$ is the number of phase leveis.

Generally, TWTA's suffer from two principal forms of distortion. The first is due to nonlinear input/output power characteristics, which causes amplitude compression with increasing input power and it is referred to as amplitude-to-amplitude (AM/AM) modulation distortion. The second distortion occurs as a result of nonlinear variation of the phase shift through the tube as the input power is changed and it is referred to as amplitude-to-phase (AM/PM) modulation distortion [1]. Thus, the transmitted signal, $s(t)$, has the form

$$
s(t)=\operatorname{Re}\left\{g(A)\left[e^{j\left(\omega_{c} t+\theta_{a}(t)-\varphi(A)\right)}\right]\right\}
$$

where $g(A)$ is the amplitude distortion due to the AM/AM non-linearity in actual satellite TWTAs and it is given by [2]

$$
g(A)=\left\{\begin{array}{cc}
10^{\alpha\left[\cos \left(\frac{\log \left(\frac{A}{\bar{A}}\right)}{\beta}\right)-1\right]} & A>\widetilde{A} \\
A & A \leq \widetilde{A}
\end{array}\right.
$$

and $\psi(A)$ is the phase distortion due to the AM/PM non-linearity and it is given by [3] 


$$
\psi(A)=k_{1}\left[1-e^{-\ddot{k}_{2} A}\right]+k_{3} A^{2}
$$

where $\bar{A}$ is the amplitude level that causes saturation.

$\widetilde{A}, \alpha_{1}$ and $\beta$ are characterizing constants of the tube [2]

$k_{1}, k_{2}$, and $k_{3}$ are constants evaluated through curve fitting of the measured characteristics.

Let us denote $g(A)$ and $\psi(A)$ by $G$ and $\Theta$ respectively. Thus, the transmitted signal, $s(t)$, has the form

$$
s(t)=\operatorname{Re}\left\{G\left[e^{j\left(\omega_{c} t+\theta_{a}(t)+\Theta\right)}\right]\right\}
$$

When the signal, $s(t)$, is transmitted, the received signal, $r(t)$, has the form

$$
\begin{aligned}
r(t) & =F s(t)+w(t) \\
& =\operatorname{Re}\left\{G \eta\left[e^{j\left(\omega_{c} t+\theta_{a}(t)+\Theta-\theta_{F}\right)}\right]\right\}+w(t)
\end{aligned}
$$

where $F$ is the interference due to slow fading and shadowing $\left(F=\eta e^{-j \theta_{F}}\right)[4]$, and $w(t)$ is an AWGN with power spectral density of $N_{0} / 2$.

\section{Proposed Classifier}

The proposed classifier consists of three main stages; 1) Preprocessing stage, 2) key features extraction stage, and 3) Classification stage. The structure of the proposed classifier is depicted in Fig. 1.

A) Preprocessing stage

In this stage, the instantaneous phase of the received signal is computed. The signal $s(t)$ contains the phase information that can be extracted by means of IQ techniques or by using discrete Hilbert transform. The extracted phase can be represented as

$$
\varphi_{\sigma}(i)=\theta_{\sigma}(i)+v(i) ;-\infty<i<\infty,-\pi \leq \varphi_{\sigma}(i)<\pi
$$

where $v(i)$ is the random phase attributed to the noise at the receiver input, $w(t)$, channel impairments, and any other measurement errors.

B) Feature extraction stage With equally likely M phases, the histogram of pure MPSK signal is given by

$$
H_{\Theta_{\theta}}(\theta)=\frac{1}{2^{\alpha}} \sum_{m=1}^{2^{a}} \delta\left(\theta-\eta_{m}(\alpha)\right)
$$

where $\eta_{m}(\alpha)$ is the phase of the $m^{\text {th }}$ phase states and can be expressed as

$$
\eta_{m}(\alpha)=\frac{\left(2 m-2^{\alpha}-1\right)}{2^{\alpha}} ; k=1,2, \ldots, 2^{\alpha}, \alpha=1,2, \ldots, \log 2 M
$$


The phase histograms of PSK2, PSK4, and PSK8 signals are shown in Fig. 2. It can be shown that for the same frame length, the amplitudes of the phase histogram of PSK2 signal is twice that of PSK4 signal and is four times that of PSK8 signal. Thus, it is clear that the magnitude of the phase histogram can be used as a feature to classify MPSK signals.

The histogram of the received signal phase is a discrete approximation to the probability density function $p d f$ of the received phase [5], that is

$$
H_{\Phi_{a}}\left(\varphi_{\alpha}\right)=\frac{F_{\Phi_{a}}\left(\varphi_{a}(i)\right)-F_{\Phi_{a}}\left(\varphi_{a}(i-1)\right)}{\varphi_{a}(i)-\varphi_{a}(i-1)} ; \varphi_{a}(i-1)<\varphi_{a}<\varphi_{a}(i), \quad i=1, \ldots, k
$$

where $k$ is the number of subintervals in the interval $\left[-\pi, \pi\left[\right.\right.$ and $F_{\Phi_{\alpha}}\left(\varphi_{\alpha}\right)$ is the cumulative distribution function of the received phase, $\varphi_{a}$, and it is calculated as

$$
F_{\Phi_{a}}\left(\varphi_{a}\right)=\int_{-\pi}^{\varphi_{a}} f_{\phi_{a}}(y) d y
$$

where $f_{\Phi_{d}}(y)$ is the pdf of the phase $\varphi_{a}$ and it is given by [6]

$$
f_{\Phi_{a}}(y)=\frac{1}{2^{\alpha}} \sum_{m=1}^{2^{a}} \frac{\exp \left[2 \gamma \cos \left(y-\eta_{m}(\alpha)\right)\right]}{2 \pi I_{0}(2 \gamma)} \quad-\pi<y<\pi
$$

where $\gamma$ is the SIR and $I_{0}[$.$] is the zero-order modified Bessel function of the first kind$ Thus, the cumulative distribution function of the received phase, $\varphi_{a}$ is given by (see appendix A)

$$
\begin{aligned}
F_{\Phi_{\omega}}\left(\varphi_{\alpha}\right)= & \frac{1}{2^{(\alpha+1)} \pi I_{0}(2 \gamma)} \sum_{m=1}^{2^{\circ}}\left[\left(\pi+\varphi_{\omega}\right) l(2 \gamma)\right. \\
& \left.+\sum_{n=1}^{\infty} \frac{2 I_{n}(2 \gamma)}{n}\left(\sin \left(n\left(\pi+\eta_{m}(\alpha)\right)\right)-\sin \left(n\left(\eta_{n}(\alpha)-\varphi_{o}\right)\right)\right)\right]
\end{aligned}
$$

In this step, the histogram of instantaneous phase of the received signal is evaluated over 32 subintervals in the $[-\pi, \pi$ [ interval. The choice of the 32 subintervals is taken in order to achieve a minimum reasonable resolution between any two adjacent states. For example in case of a pure PSK8, the phase states appear with a separation of 4 bins, i.e. there will be 3 empty bins between any two adjacent states. Also, if the number of windows becomes larger, the adjacent bin to the real state may has a value nearer to that of the real state and considered as a state.

Dividing it by the maximum element, that is, normalizes the phase histogram

$$
\dot{H}_{\Phi_{e}}(i)=H_{\Phi_{\alpha}}(i) / \max \left[H_{\omega_{\alpha}}(i)\right]
$$

\section{C) Classification stage}

In the proposed algorithm, classification of MPSK depends on the normalized phasehistogram, $\dot{H}_{\Phi_{a}}(i)$. The maximum element in the normalized phase-histogram is compared with a predefined threshold, $T$. If the maximum element exceeds the 
threshold, it will be considered a phase level. Then, the maximum element and the two adjacent elements are nullified in order not to be counted once more. Then, the previous operation is applied to the next maximum element. Finally the decision about the number of levels is taken according to the number of levels deduced. If it equals to 2 or 3 , therefore the modulation type is PSK2. If it equals to 4 or 5 , therefore the modulation type is PSK4. If it is greater than 5, therefore the modulation type is PSK8. If else is deduced, the modulation type is unknown.

\section{Threshold Determinations and Performance Evaluation}

Threshold determination is derived from 100 realizations, each with 2048 samples, for each signal of interest at SIR of 20,15 , and $10 \mathrm{~dB}$. The normalized phase histogram of the received signal is trained along the interval $[0,1]$. The optimum threshold is the one that yields the highest average success rate over each type of interest at SIR of 20,15 , and $10 \mathrm{~dB}$.

The results associated with the determination and optimization of the threshold showed that the optimum threshold, $T$, which satisfies the highest success rate, is 0.55 . Fig. 3 shows the overall success rate over different SIRs $(20,15$, and $10 \mathrm{~dB})$ at different threshold values. The figure contains four curves: 1) the dashdotted curve represents the PSK2 type, 2) the dashed curve represents the PSK4 type, 3) the dotted curve represents the PSK8 type, and 4) the solid curve represents the overall success rate.

The evaluation of the proposed algorithm is taken from 300 different realizations each with 2048 samples. All the results associated with the evaluation of the proposed method for MPSK classification are presented in Table 1, Table 2, and Table 3 at SIR of 20,15 , and 10 respectively. From these results, it is clear that all digital modulation types of interest have been correctly classified with a success rate $>94 \%$. The developed classifier was experimented at lower value of SIR (7 dB). The results showed that at lower threshold values the developed classifier could not classify PSK2 and PSK4 with an acceptable success but gives a high success rate for PSK8 as shown in Table 4. Table 5 shows the overall success rate at different SIRs of interest. Fig. 4 shows the phase histogram of the received PSK2, PSK4, and PSK8 signals at SIR $=10 \mathrm{~dB}$, each with 2048 samples. The functional flow chart for the proposed classifier is shown in Fig. 5.

\section{Conclusions}

In this paper, an algorithm for automatic modulation recognition of satellite communication signals is presented. The algorithm took in consideration the problems facing the satellite signal as the transponder non-linearity and other channel impairments such as slow fading, shadowing, and noise. The proposed algorithm showed good classification results and a robust behavior against the mentioned impairments. We have successfully solved the problem for 3 different modulation types. The proposed algorithm also showed a better performance than some classifiers that uses the same concept, Phase Based Classifiers (PBC), such 
as [7], [8], [9], and [10] (it is worth noting that these classifiers had taken in consideration only one channel impairment which is the additive white Gaussian noise) that achieve a success rate $>90 \%$ at signal-to-noise ratio (SNR) of $40 \mathrm{~dB}, 18$ $\mathrm{dB}, 15 \mathrm{~dB}$, and $10 \mathrm{~dB}$ respectively. The algorithm seems easily extendable to include higher levels (probably with some small modifications of the feature extraction and the classification stages).

\section{Appendix A}

Let the $p d f$ of the received phase, $\varphi_{a}$, is given by [6]

$$
f_{\omega_{-}}(y)=\frac{1}{2^{\alpha}} \sum_{m=1}^{z^{*}} \frac{\exp \left[2 \gamma \cos \left(y-\eta_{m}(\alpha)\right)\right]}{2 \pi I_{0}(2 \gamma)} \quad-\pi \leq y<\pi
$$

where $\gamma$ is the SIR, Io[.] is the zero-order modified Bessel function of the first kind, and $\eta_{m}(a)$ is the phase of the $m^{\text {th }}$ phase states and can be expressed as

$$
\eta_{m}(\alpha)=\frac{\left(2 m-2^{\alpha}-1\right)}{2^{\alpha}} ; \quad m=1,2, \ldots, 2^{\alpha},
$$

The cumulative distribution function of the received phase, $\varphi_{\alpha}$, is calculated as

$$
F_{\Phi_{\alpha}}\left(\varphi_{\alpha}\right)=\int_{-\infty}^{\infty} f_{\omega_{\alpha}}(y) d y
$$

where $f_{\Phi_{n}}(y)$ is the pdf of the phase, $\varphi_{a}$, in equation (A.1). Therefore,

$$
F_{\Phi_{\mu}}\left(\varphi_{a}\right)=\frac{1}{2^{(\alpha+1)} \pi I_{0}(2 \gamma)} \sum_{m=1}^{2^{*}} \int_{-\pi}^{\varphi_{u}} \exp \left[2 \gamma \cos \left(y-\eta_{m}(\alpha)\right)\right] d y
$$

Expanding using Fourier series implies,

$$
\begin{aligned}
\exp \left[2 \gamma \cos \left(y-\eta_{m}(\alpha)\right)\right] & =I_{o}(2 \gamma)+2 \sum_{n=1}^{\infty} I_{n}(2 \gamma) \cos \left(n\left(y-\eta_{m}(\alpha)\right)\right) \\
= & I_{o}(2 \gamma)+2 I_{1}(2 \gamma) \cos \left(y-\eta_{m}(\alpha)\right) \\
& +2 I_{2}(2 \gamma) \cos \left(2\left(y-\eta_{m}(\alpha)\right)\right)+\ldots
\end{aligned}
$$


Therefore, the cumulative distribution function of the received MPSK signal phase is given by

$$
\begin{aligned}
F_{\Phi_{\alpha}}\left(\varphi_{a}\right)=\frac{1}{2^{(\alpha+1)} \pi I_{0}(2 \gamma)} \sum_{m=1}^{2^{a}} & {\left[\left(\pi+\varphi_{a}\right) I_{0}(2 \gamma)\right.} \\
& \left.+\sum_{n=1}^{\infty} \frac{2 I_{n}(2 \gamma)}{n}\left(\sin \left(n\left(\pi+\eta_{m}(\alpha)\right)\right)-\sin \left(n\left(\eta_{m}(\alpha)-\varphi_{a}\right)\right)\right)\right]
\end{aligned}
$$

\section{REFERENCES:}

[1] Michel C. J. Phillip B., \& K. Sam Shannugam, "Simulation of Communication Systems ", Plenam press, New York, 1992, pp. 147.

[2] C. M. Thomas, M. Y. Weidner, \& S. H. Durrani, " Digital Amplitude Phase Keying with M-ary Alphabets ", IEEE trans. on Communication, Vol. COM-27, No.12. December 1979, pp. 1771-1781.

[3] A. L. Berman and C. E. Mahle, "Nonlinear Phase Shift in Traveling Wave Tubes as applied to Multiple Access Communications Satellite", IEEE trans. on Communication, Vol. COM-18, February 1970, pp. 37-48.

[4] Jon G. Proakis, " Digital Communications ", New York: Mc Graw-Hill, 1960.

[5] John J. Komo, "Random Signal Analysis in Engineering Systems ", Academic Press, Inc., 1987.

[6] S. S. Soliman and Z. S. Hsue, "Signal Classification Using Statistical Moments", IEEE trans. on Communications, Vol. 40, No. 5, May 1992, pp. 908-916.

[7] L. V. Domingues et. al., "A General Approach to the Automatic Classification of Radiocommunication Signals ", Signal Processing, Vol. 22, No. 3, March 1991, pp. 239-250.

[8] F. F. Liedtke, " Computer Simulation of an Automatic Classification Procedure for Digitally Modulated Communication Signals with Unknown Parameters ", Signal Processing, Vol. 6, No. 4, August 1984, pp. 311-323.

[9] Z. S. Hsue and S. S. Soliman, "Automatic Modulation Classification Using Zero Crossings ", IEE Proc. Part F Radar Signal Process, Vol. 137, No. 6, December 1990 , pp. 459-464.

[10] Asoke K. Nandi and E.E. Azzouz, "Algorithms for Automatic Modulation Recognition of Communication Signals", IEEE trans. on Communications, Vol. 46, No. 4, April 1998, pp. 431-435. 


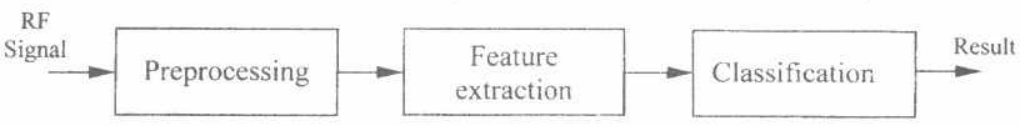

Fig. 1. Structure of the proposed classifier.

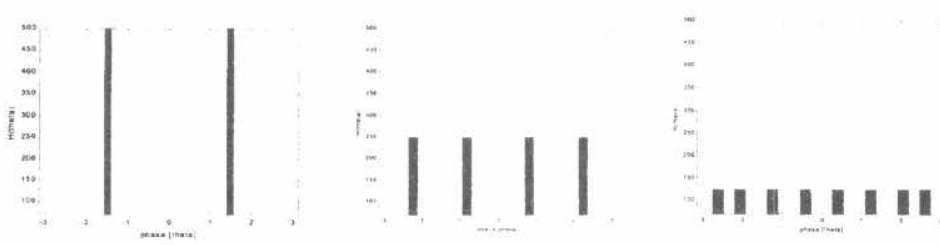

Fig. 2 Phase histogram of pure PSK2, PSK4, and PSK8 signals

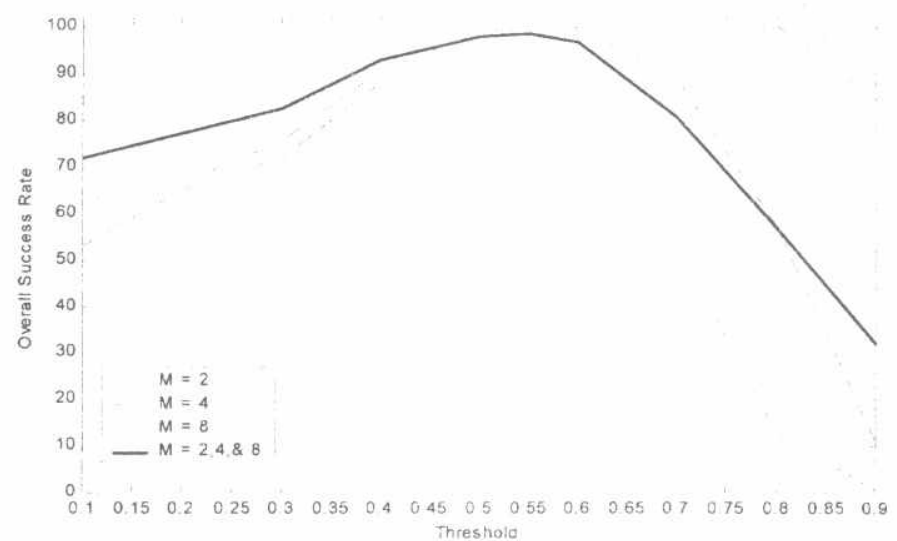

Fig. 3 Dependence of overall success rates for MPSK signals on threshold values

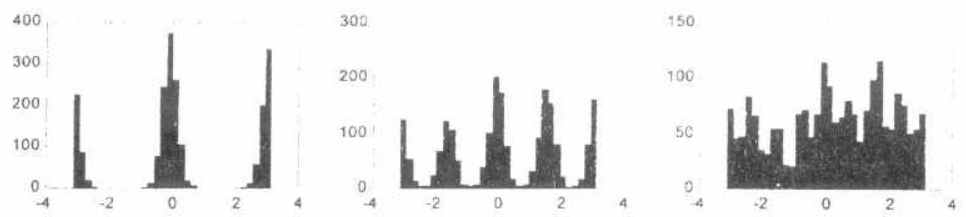

Fig. 4 Phase histogram of received PSK2, PSK4, and PSK8 signals At $\mathrm{SIR}=10 \mathrm{~dB}$. 
Instantaneous phase of the received signal, $\left\{\varphi_{\alpha}(t)\right\}$

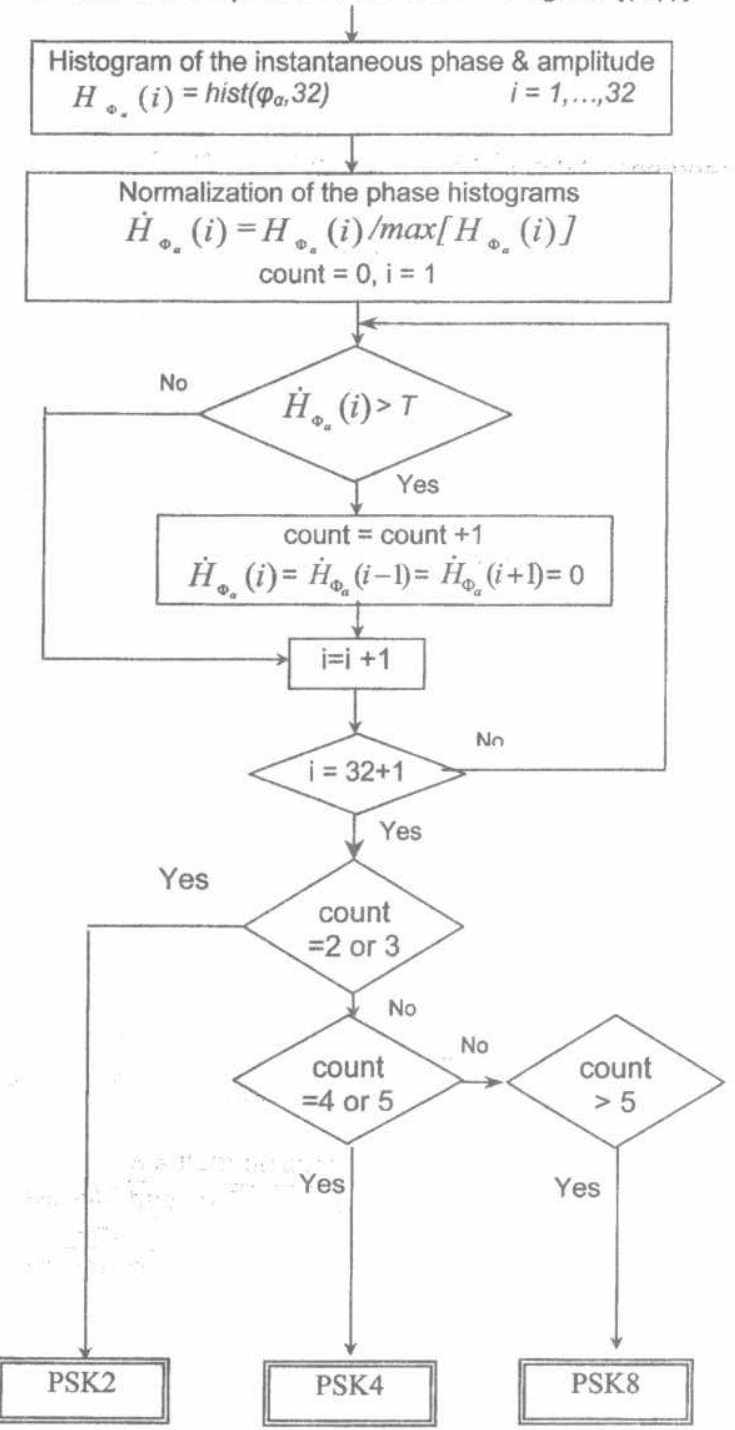

Fig. 5 The functional flow chart of the proposed algorithm 
Table 1. Confusion matrix at $\operatorname{SIR}=2.0 \mathrm{~dB}$

\begin{tabular}{|c|c|c|c|c|}
\hline \multirow{2}{*}{} & & \multicolumn{3}{|c|}{$\begin{array}{c}\text { Deduced Modulation } \\
\text { Type }\end{array}$} \\
\cline { 3 - 5 } & & PSK2 & PSK4 & PSK8 \\
\hline \multirow{2}{*}{$\begin{array}{c}\text { Simulated } \\
\text { Type }\end{array}$} & PSK2 & 100 & - & - \\
\cline { 2 - 5 } & PSK4 & - & 100 & - \\
\cline { 2 - 5 } & PSK8 & - & 1 & 99 \\
\hline
\end{tabular}

Table 2. Confusion matrix at SIR $=15 \mathrm{~dB}$

\begin{tabular}{|c|c|c|c|c|}
\hline \multirow{2}{*}{} & & \multicolumn{3}{|c|}{$\begin{array}{c}\text { Deduced Modulation } \\
\text { Type }\end{array}$} \\
\cline { 3 - 5 } & & PSK2 & PSK4 & PSK8 \\
\hline \multirow{2}{*}{$\begin{array}{c}\text { Simulated } \\
\text { Type }\end{array}$} & PSK2 & 100 & - & - \\
\cline { 2 - 5 } & PSK4 & - & 100 & - \\
\cline { 2 - 5 } & PSK8 & - & 2 & 98 \\
\hline
\end{tabular}

Table 3. Confusion matrix at SIR $=10 \mathrm{~dB}$

\begin{tabular}{|c|c|c|c|c|}
\hline \multirow{2}{*}{} & & \multicolumn{3}{|c|}{$\begin{array}{c}\text { Deduced Modulation } \\
\text { Type }\end{array}$} \\
\cline { 3 - 5 } & & PSK2 & PSK4 & PSK8 \\
\hline \multirow{2}{*}{$\begin{array}{c}\text { Simulated } \\
\text { Type }\end{array}$} & PSK2 & 99 & 1 & - \\
\cline { 2 - 5 } & PSK4 & - & 94 & 6 \\
\cline { 2 - 5 } & PSK8 & - & 2 & 98 \\
\hline
\end{tabular}

Table 4. Confusion matrix at $\mathrm{SIR}=7 \mathrm{~dB}$

\begin{tabular}{|c|c|c|c|c|}
\hline \multirow{2}{*}{} & & \multicolumn{3}{|c|}{$\begin{array}{c}\text { Deduced Modulation } \\
\text { Type }\end{array}$} \\
\cline { 3 - 5 } & & PSK2 & PSK4 & PSK8 \\
\hline \multirow{2}{*}{$\begin{array}{c}\text { Simulated } \\
\text { Type }\end{array}$} & PSK2 & 41 & 59 & - \\
\cline { 2 - 5 } & PSK4 & - & 26 & 74 \\
\cline { 2 - 5 } & PSK8 & - & - & 100 \\
\hline
\end{tabular}

Table 5. Overall success rate at different SIR

\begin{tabular}{|c|c|}
\hline SIR & $\begin{array}{c}\text { Overall Success } \\
\text { Rate (\%) }\end{array}$ \\
\hline 20 & 99.67 \\
\hline 15 & 99.33 \\
\hline 10 & 97.00 \\
\hline 9 & 88.00 \\
\hline 8 & 77.67 \\
\hline 7 & 55.67 \\
\hline
\end{tabular}

